\title{
The Relationship between Quadriceps Muscle Volume and Patellar Cartilage Morphology in Patients with Knee Osteoarthritis
}

\author{
Michael J. Berger ${ }^{1 *}$, Dean Inglis ${ }^{2}$, Charles A. McKenzie ${ }^{3}$, Kendra L. Derry ${ }^{4}$, Aashish Goela ${ }^{5}$, and \\ Timothy J. Doherty ${ }^{4,6}$ \\ ${ }^{1}$ Division of Physical Medicine \& Rehabilitation, University of British Columbia, Canada \\ ${ }^{2}$ Department of Civil Engineering, McMaster University, Canada \\ ${ }^{3}$ Department of Medical Biophysics and Robarts Research Institute, Western University, Canada \\ ${ }^{4}$ Schulich School of Medicine and Dentistry, Western University, Canada \\ ${ }^{5}$ Department of Medical Imaging, Western University, Canada \\ ${ }^{6}$ Departments of Physical Medicine and Rehabilitation, and Clinical Neurological Sciences, Western University, Canada
}

Received: June 30, 2014; Accepted: July 24, 2014; Published: August 14, 2014

*Corresponding author: Michael J Berger, Division of Physical Medicine \& Rehabilitation, University of British Columbia, London, Ontario, Canada, Tel: +1-519-685-4292 ext. 45062; Fax: +1-519-685-4098; E-mail: michael.berger@vch.ca

\begin{abstract}
The purpose of this study was to determine whether quadriceps strength and whole muscle volume or distal vastii muscle volumes (Vastus Medialis: VM, Vastus Lateralis: VL) are associated with Magnetic Resonance Imaging (MRI)-derived measures of patellar cartilage morphology in patients with knee Osteoarthritis (OA). Twenty (13 female) community dwelling participants with knee OA completed isometric maximal voluntary knee extensor contractions for determination of maximal torque. MRI using 3D multi-echo spoiled gradient echo imaging sequences and a multi-point fat-water separation method were used to acquire images of the quadriceps and the PF joint. The determinants of cartilage volume, mean cartilage thickness and maximum cartilage thickness were obtained from stepwise multiple linear regression models. A stepwise model including distal VM volume $(\beta=0.54)$ and isometric torque $(\beta=0.40)$ predicted the greatest proportion of variance in cartilage volume (adjusted $\mathrm{r}^{2}=0.75, p<0.001$. The variance in mean and maximum thickness was explained partially by distal VM volume only (predicted $31 \%$ of the variance in mean thickness and $32 \%$ of the variance in maximum thickness, $p<0.05$ ). These results provide support for further investigation of distal quadriceps function in patients with knee $\mathrm{OA}$, as distal VM volume is an important predictor of patellar cartilage morphology. Reduced distal VM volume could indicate focal weakness in this portion of the muscle that may not be evident with knee extensor strength or whole muscle volume measurement.
\end{abstract}

Keywords: Patellofemoral osteoarthritis; Cartilage morphology; Quadriceps strength; Magnetic resonance imaging

\section{Background}

Quadriceps muscle weakness leads to functional impairment $[1,2]$ and is associated with pain in patients with knee Osteoarthritis (OA) [3-5]. Quadriceps weakness may also be a modifiable risk factor for the incident and progressive structural changes that occur in knee OA [6]. Experimentally-induced muscle weakness led to degenerative changes consistent with $\mathrm{OA}$ in a rabbit model $[7,8]$, but large prospective studies in humans are equivocal as some report a significant relationship between weakness and structural disease markers [9-11], while others do not $[12,13]$. This discrepancy could be explained by a number of factors including the method for measuring structural OA (i.e. X-ray versus MRI) and the compartment affected (i.e. Tibiofemoral: TF, Versus Patellofemoral: PF). Many studies grade severity using ordinal Kellgren-Lawrence grading and measure disease progression using joint space narrowing from $\mathrm{X}$-rays. Both measures may be insensitive to subtle changes in disease onset and progression [14]. MRI provides more direct information about OA severity, as articular cartilage can be accurately and reliably quantified [15]. It is possible that the relationship between quadriceps strength and disease severity can be better characterized if quantitative measures of cartilage morphology are used to reflect disease severity.

The specific compartment affected may also influence the relationship between quadriceps strength and disease severity. In a longitudinal study, Teichtahl et al. [14] observed a protective effect for quadriceps strength on cartilage loss in the PF, but not the TF compartment [5]. It has been proposed that PFOA is a greater source of symptoms than TFOA and may constitute a different disease process altogether due to its unique biomechanics, although much less attention has been devoted to this compartment [16]. Muscle strength may play a central role in protecting the PF joint, as compression forces on this compartment are the vector sum of quadriceps muscle and patellar ligament forces [17] and the distal vastus medialis (VM) is thought to restrain lateral patellar tracking during knee flexion [18]. The angle of pennation in the VM changes distally by as much as $\sim 50-60^{\circ}$ relative to the femoral axis and these fibers are thought to stabilize the patella in the coronal 
plane [19-21]. Proximal muscle fibers would be more likely to influence movements in the sagittal plane, such as isometric knee extension. It is well known that muscle volume is the strongest predictor of isometric strength [22], however measurement of isometric strength may not adequately reflect the unique biomechanics of the distal VM and the PF joint. Hart et al. [23] reported no significant relationship between VM volume and PFOA X-ray severity, as well as no difference between the VMVastus Lateralis (VL) ratio between patients with PFOA and healthy controls. This finding could be explained by the use of whole muscle volumes for VM and VL, rather than distal vasti volumes. Indeed, there is evidence from healthy adults that distal VM cross-sectional area is independently associated with patellar cartilage morphology [24]. The purpose of this study was to further examine the relationship between muscle strength, muscle size (measured with MRI-derived measures of muscle volume) and patellar cartilage morphology in patients with knee $\mathrm{OA}$, to determine whether distal VM volume is more strongly correlated with quantitative measures of disease severity than other indices of quadriceps muscle function.

\section{Methods}

\section{Study participants}

Twenty community dwelling men and women participated in the study. Participants were included if they met the clinical criteria for knee OA outlined by the American College of Rheumatology [25], had persistent knee pain that required referral to an orthopedic surgeon and received a diagnosis of knee $\mathrm{OA}$ from the surgeon based on symptoms and radiographs [26]. Clinical disease severity was measured with the Western Ontario and McMaster Osteoarthritis Index (WOMAC, Likert Version 3.1; score range, 0-96). Anterio-posterior (for TFOA) and lateral radiographs for PFOA were graded according to KellgrenLawrence Grading (KLG) by an experienced musculoskeletal radiologist and all participants had KLG $\geq 1$ in at least one compartment. Exclusion criteria included musculoskeletal, neurological or rheumatological impairment of the lower limbs other than knee $\mathrm{OA}$, prior high tibial osteotomy, unicondylar or total knee arthroplasty or cardiopulmonary impairment that precluded performing vigorous muscle contractions. If a patient had bilateral knee $\mathrm{OA}$, the limb with the most severe symptoms (as reported by the patient), was selected as the test limb. Ethical approval for the study was obtained from the local institutional ethics review board and written consent was obtained from each participant prior to study commencement.

\section{Measurement of isometric muscle strength}

Participants were seated upright in multi-joint dynamometer (Biodex System 3, Shirley, NY), with knee and hip angles of $90^{\circ}$ and $100^{\circ}$ respectively. The centre of rotation of the knee was aligned with the axis of rotation of the dynamometer's lever arm. The force transducer was positioned with its bottom edge two fingerbreadths proximal to the medial malleolus of the test leg and fixed with a Velcro strap. A seat-belt strap was positioned across the lap in order to avoid unwanted movement. The test protocol commenced with a series of submaximal isometric contractions for the purposes of warm-up and familiarization. Participants then performed a $\sim 5 \mathrm{~s}$ isometric Maximal Voluntary Contraction (MVC) of the quadriceps. Participants were instructed to contract to attain their maximal torque output as quickly as possible and to try and maintain torque output steadily for the duration of the contraction. Three to five repetitions were performed, each separated by a minimum of $90 \mathrm{~s}$ of rest. A minimum of 3 contractions were performed and the test concluded when two consecutive MVCs differed by less than 5\%. The highest torque was recorded as the value for maximal strength. Verbal encouragement and visual feedback using the real-time digital torque was provided during all contractions to encourage maximal intensity. Torque was sampled at $100 \mathrm{~Hz}$, converted to a digital signal using a 12-bit converter (CED micro1401 mk II, Cambridge Electronic Design Limited, Cambridge, UK) and displayed in real-time using commercially available software (Spike2 ver. 5, Cambridge Electronic Design).

\section{MRI of the thigh and knee}

Images of the entire thigh and knee joint were acquired during the same session using a 3.0T MRI system (GE Discovery MR750, GE Healthcare, Waukesha, Wisc.) With an 8-coil torso phased array for the thigh and 8-coil knee phased array for the knee. Participants were positioned supine in the scanner bore. For the thigh images, a 3-plane localizing scan and calibration scan were performed to ensure the anatomy of interest was contained in the Field-Of-View (FOV). Three-dimensional (3D) axial images were acquired from proximal (femoral head) to distal (femoral condyles) with a proton density weighted multiecho-spoiled gradient echo (SPGR) imaging sequence (FOV: 42-46x21-23 cm, slice thickness: $4 \mathrm{~mm}$, matrix: $320 \times 160 \times 120$, TR: $7.4 \mathrm{~ms}$, echoes: 6 , TE, 1.4, 2.1, 2.9, 3.6, 4.4, $5.1 \mathrm{~ms}$, flip angle $5^{\circ}$, bandwidth: \pm $142.86 \mathrm{khz}$ ). An investigational version of the IDEAL (Iterative Decomposition of water and fat with Echo asymmetry and Least squares estimation) multipoint water-fat separation method that is $\mathrm{T} 1$ independent, $\mathrm{T} 2{ }^{*}$ corrected and employs accurate spectral modeling of fat, was used to reconstruct both water-only and fat-only images from the multiecho SPGR source images [27]. Fat-water separation also facilitated the exclusion of non-muscle tissue (i.e. Fat). Image analysis was performed on the water only images produced by the IDEAL reconstruction for assessment of muscle volume.

For the knee scans a 3-plane localization scan, calibration scan and 3D SPGR-FS scout scans were acquired to ensure the anatomy of interest was contained in FOV. An oblique plane 3D SPGR IDEAL imaging sequence was used (FOV: $16 \mathrm{~cm}$, slice thickness: $1 \mathrm{~mm}$, matrix 512x512, TR: $7.8 \mathrm{~ms}$, TE: 1.4, 2.2, 3.0, 3.8, 4.6 and $5.4 \mathrm{~ms}$, flip angle $3^{\circ}$, bandwidth $\pm 90.91 \mathrm{khz}$ ). Image postprocessing was performed on the water images only.

\section{Image post-processing}

A combination of manual and pixel threshold growing techniques (from a defined seedpoint) using open-source osirix image processing software (version 3.7, Geneva, Switzerland) were used to analyze images in the axial plane to calculate quadriceps muscle volume (Figure 1a). Analysis began proximally 
from the first slice not containing the gluteal muscle to the last distal slice containing the rectus femoris because it is difficult to differentiate between thigh muscles at the most proximal and distal slices [28]. Quadriceps muscle volume was calculated from the water-only images by manually outlining the most proximal and distal slices with the brush tool to create Regions of Interest (ROI). Once the quadriceps had been roughly outlined, all pixel values outside the rois were set to zero. Subsequently, a 3D threshold-growing tool was used to grow rois for the quadriceps muscle tissue only from a defined seedpoint within the muscle. This was done to ensure exclusion of non-muscular tissue in the septal spaces. Any errors produced by the automatic tool were corrected manually. The software automatically calculated volume for the series of rois. The reliability of this method has been previously established [29]. This method was also used to calculate VL volume (Figure 1b). All slices from the most distal slice containing visible fibres of the VL to the slice $\sim 4 \mathrm{~cm}$ proximal to this point were included in the analysis. Distal $(\sim 4 \mathrm{~cm}) \mathrm{VM}$ volume was calculated in an identical fashion but from the knee images (Figure 1c). The distal $\sim 4 \mathrm{~cm}$ was selected as the fibres of VM and VL deviate from the femoral axis as they approach the patella and assume a more horizontal orientation [30].

Patellar bone and cartilage were segmented with customdesigned software after transferring saved images to a workstation (Figure 2). Surface generation of the articular cartilage and sub-chondral bone surfaces was performed in a slice-by-slice manner. Due to the high contrast between bone and cartilage in our images, the sub-chondral bone surface area was segmented by means of a semi-automated edge tracking algorithm incorporating Dijkstra's shortest path algorithm. The articular cartilage surface was segmented from surrounding tissues (e.g., meniscus, ligaments, opposing cartilage etc.) By manually fitting one or more cubic splines allowing for denuded areas. End points of the articular cartilage contours were initiated and terminated on sub-chondral bone contours. The software allowed for forward or reverse propogation of contours from one slice to another to expedite the segmentation process. Upon completion of contour definition, binary voxel maps were generated from the aggregation of the areas contained between articular cartilage and sub-chondral bone contours. Cartilage volume was determined by numerical integration of segmented voxels. Mean cartilage thickness was determined by 3D Euclidean distance transformation, accounting for denuded regions by assigning a thickness value of zero. The standard deviations of mean and maximum thickness were also determined. Interrater reliability defined by the intraclass correlation coefficient (ICC, 2:1) and 95\% confidence intervals was 0.95 (0.81-0.99), 0.97 (0.87-0.99) and $0.90(0.67-0.98)$ for cartilage volume mean thickness and maximum thickness, respectively (for 10 cases).

\section{Statistics}

The strength of correlation between study parameters was assessed with Pearson's Correlation Coefficient ( $r$ ) and Spearman rank correlation coefficient for parametric and non-parametric data, respectively. Variables with significant correlations were included in stepwise multiple linear regression models with



Figure 1: A combination of manual and semi-automated segmentation techniques was used to calculate whole quadriceps muscle volume (a) and distal vastus lateralis volume from axial thigh images. Distal vastus medialis volume (c) was segmented using the same techniques from axial knee images.

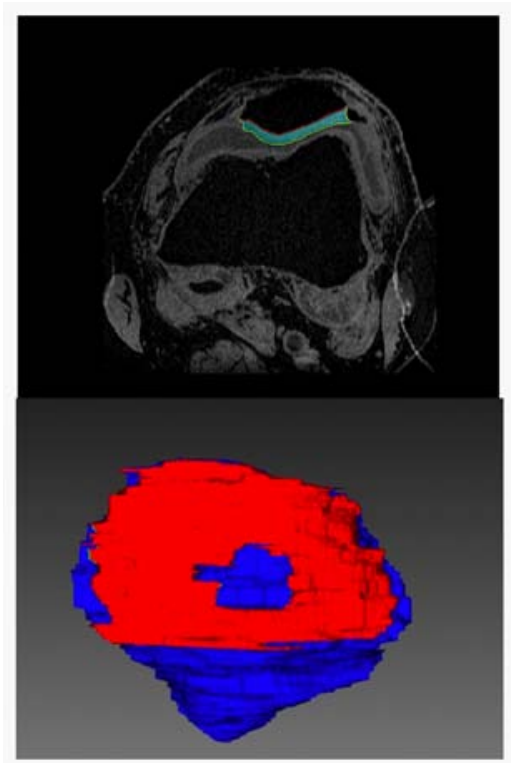

Figure 2: Axial slice of knee images showing 2D segmentation of the patellar cartilage (top panel) and in-progress 3D reconstruction of patellar cartilage on the subchondral bone surface as each new slice is segmented (bottom panel). Note denuded area of subchondral bone in the centre of the $3 \mathrm{D}$ reconstruction. 
each cartilage parameter used as an outcome variable (i.e. Three regression models for cartilage volume, mean thickness and maximum thickness). The adjusted $r^{2}$ was used to determine the strength of prediction of the entire model, $\beta$-coefficients were used to quantify the relative importance of each predictor variable to the model and tolerance values were reported to provide an index of multicollinearity between independent variables. Level of significance was established at $p<0.05$. All statistical procedures were performed with SPSS (version 17.0, Chicago, IL).

\section{Results}

Descriptive statistics, muscle strength and volume measures and cartilage morphology parameters for the study participants are presented in Table 1 . Correlation coefficients reflecting the magnitude of association between muscle/demographic variables and cartilage variables are presented in Table 2 . Variables displaying a significant association $(p<0.05)$ with cartilage outcome variables were included in stepwise multiple regression models. The results from the stepwise multiple regression models are presented in Table 3 for cartilage volume, mean thickness and maximum thickness. Tolerance values are only displayed in Table 3, as cartilage volume alone had more than one predictor variable. Generally, distal VM volume was the strongest predictor of cartilage parameters, with isometric torque predicting an additional $7 \%$ of the variance in cartilage volume (Table 3). Variables excluded from all models were sex, body mass and total quadriceps volume.

\section{Discussion}

Quadriceps muscle atrophy and weakness have been

Table 1: Study parameters.

\begin{tabular}{|c|c|}
\hline $\begin{array}{c}\text { Characteristics of the study } \\
\text { participants }(\mathbf{n}=\mathbf{2 0})\end{array}$ & $\begin{array}{c}\text { Mean } \pm \text { SD (minimum, } \\
\text { maximum) }\end{array}$ \\
\hline Sex (Male/Female) & $7 / 13$ \\
\hline Age (years) & $61.7 \pm 6.2(52,74)$ \\
\hline Height $(\mathrm{m})$ & $1.68 \pm 0.07(1.51,1.83)$ \\
\hline Body mass $(\mathrm{kg})$ & $82.4 \pm 11.8(62,109)$ \\
\hline BMI $\left(\mathrm{kg} / \mathrm{m}^{2}\right)$ & $29.4 \pm 4.6(20.7,37.3)$ \\
\hline WOMAC pain subscale & $8 \pm 4(1,15)$ \\
\hline WOMAC function subscale & $26 \pm 16(2,57)$ \\
\hline WOMAC total score & $39 \pm 22(5,80)$ \\
\hline Isometric torque $(\mathrm{N} \bullet \mathrm{m})$ & $135 \pm 66(61,283)$ \\
\hline Quadriceps muscle volume $\left(\mathrm{cm}^{3}\right)$ & $774 \pm 293(454,1424)$ \\
\hline Distal VM volume $\left(\mathrm{cm}^{3}\right)$ & $20.9 \pm 6.5(11.9,35.0)$ \\
\hline Distal VL volume $\left(\mathrm{cm}^{3}\right)$ & $13.5 \pm 3.7(8.2,22.9)$ \\
\hline Cartilage volume $\left(\mathrm{mm}^{3}\right)$ & $3700 \pm 1216(2201,6305)$ \\
\hline Mean thickness $\left(\mathrm{cm}^{3}\right)$ & $1.66 \pm 0.47(0.93,2.57)$ \\
\hline Maximum thickness $\left(\mathrm{cm}^{3}\right)$ & $5.01 \pm 1.04(3.00,7.00)$ \\
\hline
\end{tabular}

SD: Standard Deviation; BMI: body mass index; WOMAC: Western Ontario and McMaster Osteoarthritis Index; VM: Vastus Medialis; VL: Vastus Lateralis
Table 2: Correlation coefficients between cartilage parameters and demographic and muscle parameters.

\begin{tabular}{|l|c|c|c|}
\hline & $\begin{array}{c}\text { Cartilage } \\
\text { volume }\end{array}$ & $\begin{array}{c}\text { Mean } \\
\text { thickness }\end{array}$ & $\begin{array}{c}\text { Maximum } \\
\text { thickness }\end{array}$ \\
\hline Sex & $0.70^{* *}$ & $0.50^{*}$ & 0.39 \\
\hline Body mass & $0.49^{*}$ & 0.33 & 0.34 \\
\hline Isometric torque & $0.81^{* * *}$ & $0.60^{* *}$ & $0.50^{*}$ \\
\hline $\begin{array}{l}\text { Quadriceps muscle } \\
\text { volume }\end{array}$ & $0.79^{* * *}$ & $0.48^{*}$ & $0.59^{* *}$ \\
\hline Distal VM volume & $0.83^{* * *}$ & $0.58^{* *}$ & $0.59^{* *}$ \\
\hline Distal VL volume & 0.43 & 0.24 & 0.05 \\
\hline
\end{tabular}

SD: Standard Deviation; WOMAC: Western Ontario and McMaster Osteoarthritis Index; VM: Vastus Medialis; VL: Vastus Lateralis. ${ }^{*} p<0.05$, ${ }^{* *} p<0.01,{ }^{* * *} p<0.001$

: Determinants of cartilage volume, mean thickness and maximum thickness using stepwise multiple regression.

\begin{tabular}{|c|c|c|c|}
\hline $\begin{array}{c}\text { parameters included in the } \\
\text { model }\end{array}$ & $\boldsymbol{\beta}$-coeffcient & adjusted $\mathbf{r}^{2}$ & tolerance \\
\hline cartilage volume & & & \\
\hline model 1 & & $0.68^{*}$ & \\
\hline distal VM volume & 0.83 & & \\
\hline model 2 & & $0.75^{*}$ & \\
\hline distal VM volume & 0.55 & & 0.49 \\
\hline isometric torque & 0.40 & & 0.49 \\
\hline mean thickness & & & \\
\hline model 1 & & $0.31^{*}$ & \\
\hline distal VM volume & 0.59 & & \\
\hline maximum thickness & & & \\
\hline model 1 & & $0.32^{*}$ & \\
\hline distal VM volume & 0.59 & & \\
\hline
\end{tabular}

VM: Vastus Medialis; VL: Vastus Lateralis. Variables excluded: 1) cartilage volume: sex, body mass, quadriceps volume; 2) mean thickness: quadriceps volume, isometric torque; 3 ) maximum thickness: quadriceps volume, isometric torque ${ }^{*} p<0.001$

implicated in the etiology and pathogenesis of knee OA, although strong epidemiological associations have not been uniformly reported [9-11,13,31]. We hypothesized that inter-study variability may be due to the method of quantifying disease severity and the joint under consideration (PF vs. TF). When considering the biomechanics of the PF joint, it is possible that the magnitude of isometric knee extensor torque and whole muscle volume do not influence loading at the PF joint to same extent as the more horizontally oriented distal VM fibres. The results of our study support this concept as distal VM volume was the strongest predictor among muscle parameters of patellar cartilage morphology in this patient sample. This has implications toward explaining the variability observed in previous studies and to understanding the mechanisms of cartilage degradation in PFOA.

Few studies have assessed the relationship between quadriceps muscle strength and OA severity using quantitative 
MRI assessment. It has been shown previously that X-ray indices of PFOA disease severity show poor agreement with PF defects visualized with knee arthroscopy [32]. Quantitative assessment of cartilage morphology using MRI has been shown to be a valid and responsive measure in longitudinal analysis of cartilage in healthy individuals and those with OA [15]. Our observation that patellar cartilage morphology is associated with distal VM volume builds on previous studies reporting a relationship between quadriceps strength and size measures and PF cartilage morphology. In a longitudinal analysis of cartilage morphology using semi-quantitative assessment, Amin et al. [5] found that reduced quadriceps strength was independently associated with increased lateral PF cartilage loss in patients with knee OA (although no association was noted between strength and TF cartilage). Furthermore, it has previously been reported that distal VM CSA, but not VL CSA is positively correlated with patellar cartilage volume in a healthy middle-aged population [33]. Conversely, Hudlemaier et al. [34] observed a weak association between mid-thigh quadriceps CSA and patellar cartilage volume and thickness in healthy subjects. Furthermore, Hart et al. [23] reported that whole muscle VM volume was not associated with PFOA severity. Our results further understanding of this relationship as stepwise multiple regression ensured that only those variables that contributed significantly to the model were included. For cartilage volume, we observed that distal VM volume explained a large proportion of the variance, while the inclusion of isometric torque in the model explained only an additional 7\% (Table 3). For mean and maximum cartilage thickness, distal VM volume was the only variable included in the model (Table 3). The importance of distal VM volume to patellar cartilage morphology over other quadriceps variables could explain the variability observed in studies examining the relationship between disease severity and knee OA.

The relationship between distal VM volumes to patellar cartilage morphology has implications toward understanding the etiology and pathogenesis of PFOA. Muscle atrophy due to disuse has been postulated to be a primary mechanism of muscle weakness in knee $\mathrm{OA}$ [35], but whether atrophy is uniform throughout the entire muscle or restricted to a specific location has yet to be considered. Although muscle atrophy can be quantified only in a longitudinal design, our finding that distal VM volume was highly predictive of cartilage volume (and to a lesser extent mean and maximum thickness) suggests that VM morphology could have an impact on PF function, or vice versa. An intact distal VM is necessary to ensure proper patellar tracking and weakness or muscle imbalances can lead to increasing lateral PF contact pressure [18]. Lower distal VM volume could be indicative of reduced strength in this portion of the muscle alone, independent of reductions in strength elsewhere in the muscle. For example, Pattyn et al. [36] reported that VM obliqus CSA was reduced in a group with patellofemoral pain syndrome versus a control group, while mid-thigh quadriceps CSA was similar between groups. Due to the oblique orientation of its muscle fibres, the line of action of the distal VM is relatively horizontal compared to the proximal quadriceps muscle fibres, therefore weakness localized to this portion of the muscle may not be apparent when measuring isometric or dynamic knee extensor strength. Accordingly, this could explain why distal VM volume was a stronger predictor of patellar cartilage volume than isometric torque or quadriceps volume. In support of this, it has been reported that the oblique fibres of the distal VM do not participate significantly in knee extension [37]. Alternatively, it is possible that the temporal association between distal VM volume and PFOA is such that a painful PF compartment results in atrophy of the distal quadriceps. Due to the cross-sectional design employed in this study, it is not possible to determine whether atrophy precedes or follows OA changes. This can only be determined in a longitudinal design.

Along with distal VM atrophy, there are other potential mechanisms of distal VM dysfunction that could be associated with altered $\mathrm{PF}$ biomechanics and structural damage to this compartment. We reported previously that motor unit recruitment is altered and firing rates are reduced in the distal VM of knee OA subjects compared to healthy controls [38]. Further to this point, muscle quality may also be altered in this muscle as Fink et al. [39] reported type II fibre atrophy and fibre type grouping (consistent with a collateral reinnervation process) in the VM of peri-arthroplasty knee OA patients. Changes in surface electromyographic activity of the distal VM have not been observed in patients with symptomatic knee OA [40] and it is unclear if neuromuscular alterations in this portion of the muscle influence PF biomechanics.

There are several limitations in interpreting the results of this study. First, the low sample size reduces the external validity of the results and the current findings require confirmation in a larger study sample. Due to the length of the MRI scan required to obtain an adequate signal-to-noise ratio and resolution for both knee and thigh images ( $\sim 1$ hour), combined with the time required for segmentation of the relevant structures it was necessary to restrict the sample size. Further to the small sample size, we did not possess the necessary study power to analyze men and women separately. It has been reported that women with knee OA experience a greater rate of patellar cartilage loss than men [41]. Furthermore, PF biomechanics are different between sexes with women having a larger Q-angle (angle formed between the patellar tendon and the resultant line of force of the quadriceps muscles) resulting in increased lateral $\mathrm{PF}$ contact pressures [16]. As mentioned previously, due to the cross-sectional design of this study, we were not able to quantify muscle atrophy, nor were we able to compare VM volume of the OA participants to a control group without OA. Last, only cartilage volume was assessed in this study. MRI is useful for detecting and quantifying other pathological changes associated with OA such as osteophytosis, bone marrow lesions, synovial thickening etc., some of which may be better correlated with OA symptoms than cartilage morphology [15].

\section{Conclusion}

We report that distal VM volume was the primary predictor of cartilage morphological features in a sample of participants with symptomatic and radiographic knee OA. As distal VM function has been shown to be integral to proper PF joint biomechanics, it is 
possible that weakness specific to this portion of the quadriceps muscle contributes to the patellar cartilage degradation, or alternatively that a painful knee joint results in reduced distal VM volume. As these results are preliminary and were derived from a small study sample, further cross-sectional and longitudinal analysis is required to determine the relationship between the pathological features of PFOA and distal VM function.

\section{References}

1. Hurley MV, Scott DL. Improvements in quadriceps sensorimotor function and disability of patients with knee osteoarthritis following a clinically practicable exercise regime. Br J Rheumatol. 1998; 37(11):1181-1187.

2. Hurley MV, Scott DL, Rees J, Newham DJ. Sensorimotor changes and functional performance in patients with knee osteoarthritis. Ann Rheum Dis. 1997; (56):641-648.

3. O’Reilly SC, Jones A, Muir KR, Doherty M. Quadriceps weakness in knee osteoarthritis: the effect on pain and disability. Ann Rheum Dis. 1998; 57(10):588-594.

4. Hall MC, Mockett SP, Doherty M. Relative impact of radiographic osteoarthritis and pain on quadriceps strength, proprioception, static postural sway and lower limb function. Ann Rheum Dis 2006; 65(7):865-870.

5. Amin S, Baker K, Niu J, Clancy M, Goggins J, Guermazi A, et al. Quadriceps strength and the risk of cartilage loss and symptom progression in knee osteoarthritis. Arthritis Rheum 2009; 60(1):189-198.

6. Felson DT. Risk factors for osteoarthritis: understanding joint vulnerability. Clin Orthop Relat Res. 2004; S16-21.

7. Rehan Youssef A, Longino D, Seerattan R, Leonard T, Herzog W. Muscle weakness causes joint degeneration in rabbits. Osteoarthritis Cartilage. 2009; 17(9):1228-1235.

8. Longino D, Frank C, Herzog W. Acute botulinum toxin-induced muscle weakness in the anterior cruciate ligament-deficient rabbit. J Orthop Res. 2005; 23(6):1404-1410.

9. Baker KR, Xu L, Zhang Y, Nevitt M, Niu J, Aliabadi P, et al. Quadriceps weakness and its relationship to tibiofemoral and patellofemoral knee osteoarthritis in Chinese: the Beijing osteoarthritis study. Arthritis Rheum. 2004; 50(6):1815-1821.

10. Slemenda C, Heilman DK, Brandt KD, Katz BP, Mazzuca SA, Braunstein $\mathrm{EM}$, et al. Reduced quadriceps strength relative to body weight: a risk factor for knee osteoarthritis in women? Arthritis Rheum. 1998; 41(11):1951-1959.

11. Segal NA, Glass NA, Torner J, Yang M, Felson DT, Sharma L, et al. Quadriceps weakness predicts risk for knee joint space narrowing in women in the MOST cohort. Osteoarthritis Cartilage. 2010; 18(6):769775. doi: 10.1016/j.joca.2010.02.002.

12. Segal NA, Torner JC, Felson D, Niu J, Sharma L, Lewis CE, et al. Effect of thigh strength on incident radiographic and symptomatic knee osteoarthritis in a longitudinal cohort. Arthritis Rheum. 2009; 61(9):1210-1217. doi: 10.1002/art.24541.

13. Brandt KD, Heilman DK, Slemenda C, Katz BP, Mazzuca SA, Braunstein EM, et al. Quadriceps strength in women with radiographically progressive osteoarthritis of the knee and those with stable radiographic changes. J Rheumatol. 1999; 26(11):2431-2437.

14. Teichtahl AJ, Wluka AE, Davies-Tuck ML, Cicuttini FM. Imaging of knee osteoarthritis. Best Pract Res Clin Rheumatol. 2008; 22: 1061-1074. doi: 10.1016/j.berh.2008.09.004.
15. Eckstein F, Burstein D, Link TM. Quantitative MRI of cartilage and bone: degenerative changes in osteoarthritis. NMR Biomed 2006; $19(7): 822-854$.

16. Hinman RS, Crossley KM. Patellofemoral joint osteoarthritis: an important subgroup of knee osteoarthritis. Rheumatology (Oxford) 2007; 46(7):1057-1062.

17. Hehne HJ. Biomechanics of the patellofemoral joint and its clinical relevance. Clin Orthop Relat Res. 1990; (258): 73-85.

18. Sakai N, Luo ZP, Rand JA, An KN. The influence of weakness in the vastus medialis oblique muscle on the patellofemoral joint: an in vitro biomechanical study. Clin Biomech (Bristol, Avon). 2000; 15(5):335339.

19. Hubbard JK, Sampson HW, Elledge JR. The vastus medialis oblique muscle and its relationship to patellofemoral joint deterioration in human cadavers. J Orthop Sports Phys Ther 1998; 28(6):384-391.

20. Hubbard JK, Sampson HW, Elledge JR. Prevalence and morphology of the vastus medialis oblique muscle in human cadavers. Anat Rec. 1997; 249 (1):135-142.

21. Peeler J, Cooper J, Porter MM, Thliveris JA, Anderson JE. Structural parameters of the vastus medialis muscle. Clin Anat. 2005;18 (4):281289.

22. Doherty TJ. Invited review: Aging and sarcopenia. J Appl Physiol. 2003; 95(4):1717-1727.

23. Hart HF, Ackland DC, Pandy MG, Crossley KM. Quadriceps volumes are reduced in people with patellofemoral joint osteoarthritis. Osteoarthritis Cartilage. 2012; 20(8):863-868. doi: 10.1016/j. joca.2012.04.009.

24. Berry PA, Teichtahl AJ, Galevska-Dimitrovska A, Hanna FS, Wluka $\mathrm{AE}$, Wang $\mathrm{Y}$, et al. Vastus medialis cross-sectional area is positively associated with patella cartilage and bone volumes in a pain-free community-based population. Arthritis Res Ther. 2008; 10(6):R143.

25. Altman R, Asch E, Bloch D, Bole G, Borenstein D, Brandt K, et al. Development of criteria for the classification and reporting of osteoarthritis. Classification of osteoarthritis of the knee. Diagnostic and Therapeutic Criteria Committee of the American Rheumatism Association. Arthritis Rheum. 1986; 29(8):1039-1049.

26. Gossec L, Paternotte S, Maillefert JF, Combescure C, Conaghan PG, Davis AM, et al. The role of pain and functional impairment in the decision to recommend total joint replacement in hip and knee osteoarthritis: an international cross-sectional study of 1909 patients. Report of the OARSI-OMERACT Task Force on total joint replacement. Osteoarthritis Cartilage. 2011; 19(2):147-154. doi: 10.1016/j. joca.2010.10.025.

27. Reeder SB, McKenzie CA, Pineda AR, Yu H, Shimakawa A, Brau AC, et al. Water-fat separation with IDEAL gradient-echo imaging. J Magn Reson Imaging. 2007; 25(3):644-652.

28. Cotofana S, Hudelmaier M, Wirth W, Himmer M, Ring-Dimitriou S, Sanger AM, et al. Correlation between single-slice muscle anatomical cross-sectional area and muscle volume in thigh extensors, flexors and adductors of perimenopausal women. Eur J Appl Physiol 2010; 110(1):91-97. doi: 10.1007/s00421-010-1477-8.

29. Berger MJ, McKenzie CA, Chess DG, Goeala A, Doherty TJ. Sex Differences in Quadriceps Strength in OA. Int J Sports Med. 2012; 33(11):926-33. doi: 10.1055/s-0032-1311587.

30. Waligora AC, Johanson NA, Hirsch BE. Clinical anatomy of the quadriceps femoris and extensor apparatus of the knee. Clin Orthop 
Relat Res. 2009; 467(12):3297-3306. doi: 10.1007/s11999-0091052-y

31. Segal NA, Glass NA, Felson DT, Hurley M, Yang M, Nevitt M, et al. Effect of quadriceps strength and proprioception on risk for knee osteoarthritis. Med Sci Sports Exerc. 2010; 42(11):2081-2088.

32. Chang CB, Seong SC, Kim TK. Evaluations of radiographic joint space-do they adequately predict cartilage conditions in the patellofemoral joint of the patients undergoing total knee arthroplasty for advanced knee osteoarthritis? Osteoarthritis Cartilage. 2008(10); 16(10):11601166.

33. Berry PA, Hanna FS, Teichtahl AJ, Wluka AE, Urquhart DM, Bell RJ, et al. Vastus medialis cross-sectional area is associated with patella cartilage defects and bone volume in healthy women. Osteoarthritis Cartilage. 2008; 16(8):956-960. doi: 10.1016/j.joca.2007.11.011.

34. Hudelmaier M, Glaser C, Englmeier KH, Reiser M, Putz R, Eckstein F. Correlation of knee-joint cartilage morphology with muscle crosssectional areas vs. anthropometric variables. Anat Rec A Discov Mol Cell Evol Biol. 2003; 270(2):175-184.

35. Berger MJ, Doherty TJ. Sarcopenia: Prevalence, Mechanisms, and Functional Consequences. Interdiscip Top Gerontol. 2010; 37:94-114. doi: 10.1159/000319997.
36. Pattyn E, Verdonk P, Steyaert A, Vanden Bossche L, Van den Broecke W, Thijs Y, et al. Vastus medialis obliquus atrophy: does it exist in patellofemoral pain syndrome? Am J Sports Med. 2011; 39(7):14501455.

37. Goh JC, Lee PY, Bose K. A cadaver study of the function of the oblique part of vastus medialis. J Bone Joint Surg Br. 1995; 77(2):225-231.

38. Berger MJ, Chess DG, Doherty TJ. Vastus medialis motor unit properties in knee osteoarthritis. BMC Musculoskelet Disord. 2011; 12:199. doi: 10.1186/1471-2474-12-199.

39. Fink B, Egl M, Singer J, Fuerst M, Bubenheim M, Neuen-Jacob E. Morphologic changes in the vastus medialis muscle in patients with osteoarthritis of the knee. Arthritis Rheum. 2007; 56(11):3626-3633.

40. Hinman RS, Bennell KL, Metcalf BR, Crossley KM. Temporal activity of the vastus medialis obliquus and vastus lateralis in symptomatic knee osteoarthritis. Am J Phys Med Rehabil. 2002; 81(9):684-690.

41. Cicuttini F, Wluka A, Wang Y, Stuckey S. The determinants of change in patella cartilage volume in osteoarthritic knees. J Rheumatol. 2002; 29(12):2615-2619. 